\title{
Noether Current of the Surface Term of Einstein-Hilbert Action, Virasoro Algebra, and Entropy
}

\author{
Bibhas Ranjan Majhi \\ IUCAA, Ganeshkhind, Pune University Campus, Post Bag 4, Pune 411 007, India \\ Correspondence should be addressed to Bibhas Ranjan Majhi; bibhas@iucaa.ernet.in
}

Received 30 April 2013; Accepted 28 June 2013

Academic Editor: Mauricio Bellini

Copyright (C) 2013 Bibhas Ranjan Majhi. This is an open access article distributed under the Creative Commons Attribution License, which permits unrestricted use, distribution, and reproduction in any medium, provided the original work is properly cited.

\begin{abstract}
A derivation of Noether current from the surface term of Einstein-Hilbert action is given. We show that the corresponding charge, calculated on the horizon, is related to the Bekenstein-Hawking entropy. Also using the charge, the same entropy is found based on the Virasoro algebra and Cardy formula approach. In this approach, the relevant diffeomorphisms are found by imposing a very simple physical argument: diffeomorphisms keep the horizon structure invariant. This complements similar earlier results (Majhi and Padmanabhan (2012)) (arXiv:1204.1422) obtained from York-Gibbons-Hawking surface term. Finally we discuss the technical simplicities and improvements over the earlier attempts and also various important physical implications.
\end{abstract}

\section{Introduction}

The thermodynamic properties of horizon arise from the combination of the general theory of relativity and the quantum field theory. This was first observed in the case of black holes $[1,2]$. Now it is evident that it is much more general, and a local Rindler observer can attribute temperature and entropy to the null surfaces in the context of the emergent paradigm of gravity [3-6]. Such a generality might provide us a deeper insight into the quantum nature of the spacetime. So far several attempts have been made to know the microscopic origin of the entropy, but every method has its own merits and demerits. Among others, Carlip made an attempt $[7,8]$ in the context of Virasoro algebra to illuminate this aspect which is basically the generalisation of the method by Brown and Henneaux [9]. In brief, in this method one first defines a bracket among the Noether charges and calculate it for certain diffeomorphisms, chosen by some physical considerations. It turns out that the algebra is identical to the Virasoro algebra. The central charge and the zero mode eigenvalue of the Fourier modes of the charge are then automatically identified in which after substituting in the Cardy formula [10-12] one finds the Bekenstein-Hawking entropy. (For a complete list of works which lead to further development of this method, see [13-53].) In all the previous attempts, the Noether current was taken in relation to the Einstein-Hilbert
$(\mathrm{EH})$ action, and the analysis was on shell; that is, equation of motion has been used explicitly. Later an off shell analysis and a generalization to Lanczos-Lovelock gravity have been presented in [54].

Earlier [55], based on the Virasoro algebra approach, we showed that the entropy can also be obtained from the Noether current corresponding to the York-Gibbons-Hawking surface term. But it is not clear if the same can be achieved from the surface term of the Einstein-Hilbert action since they are not exactly identical. So it is necessary to investigate this issue in the light of Virasoro algebra context, particullary because both surface terms lead to the same entropy on the horizon. This will complement our earlier work [55].

In this paper we will use the Noether current associated with the surface term of $\mathrm{EH}$ action. Before going into the motivations for taking the surface term only, let us first highlight some peculiar facts of $\mathrm{EH}$ action which are essential for the present purpose.

(i) It is an unavoidable fact that to obtain the equation of motion in the Lagrangian formalism one has to impose some extra prescription, like adding extra boundary term (in this case York-Gibbons-Hawking term). This is because the action contains secondorder derivative of metric tensor $g_{a b}$. But unfortunately the choice of the surface term is not unique. 
This is quite different from other well-known field theories.

(ii) The EH action can be separated into two terms: one contains the squires of the Christoffel connections (i.e., it is in ГГ-ГГ structure) and the other one contains the total derivation of $\Gamma(\partial Г-\partial Г$ structure). We will call them $L_{\text {quad }}$ and $L_{\text {sur }}$, respectively. Interestingly, Einstein's equation of motion can be obtained solely from $L_{\text {quad }}$ by using the usual variation principle where no additional prescription is required [56].

(iii) The most important one is that these two terms are related by an algebraic relation, usually known as holographic relation $[57,58]$.

Interestingly, all the previous features happened to be common even for the Lanczos-Lovelock theory [59]. For a recent review in this direction, see [60].

Although an extensive study on the Noether current of gravity has been done starting from Wald et al. [61-63], discussion on the current derived from $L_{\text {sur }}$ is still lacking. To motivate why one should be interested, let us summarise the already observed facts as follows.

(i) It is expected that the entropy is associated with the degrees of freedom around or on the relevant null surface rather than the bulk geometry of spacetime.

(ii) This surface term calculated on the Rindler horizon gives exactly the Bekenstein-Hawking entropy [56].

(iii) Extremization of the surface term with respect to the diffeomorphism parameter whose norm is a constant leads to Einstein's equation [57].

(iv) Another interesting fact is that, in a small region around an event, $\mathrm{EH}$ action reduces to a pure surface term when evaluated in the Riemann normal coordinates.

All these indicate that either the bulk and the surface terms are duplicating all the information or the actual dynamics is stored in surface term rather than in bulk term. To illuminate more on this issue, one needs to study every aspect of the surface term.

In this paper, we will discuss the Noether realization of the surface term of the $\mathrm{EH}$ action; particularly we will examine if the Noether current represents the Virasoro algebra for a certain class of diffeomorphisms. This is necessary to have a deeper understanding of the role of the surface term in the gravity. Also it will give a further insight into the earlier claim: the actual information of the gravity is stored in the surface. To do this explicitly, we will consider the form of the metric close to the null surface in the local Rindler frame around some event. This is given by the Rindler metric. The reasons for choosing such metric are as follows. According to equivalence principle, gravity can be mimicked by an accelerated observer, and an uniformly accelerated frame will have Rindler metric. Apart from that, it is a relevant frame for an observer sitting very near to the black hole horizon. Hence any thermodynamic feature of the null surface can be attributed by this metric, and it provides a general description which was originally obtained only for the black hole horizon. Moreover, all the quantities will be observer dependent.

In this paper we will proceed as follows. First a detailed derivation of the Noether current for a diffeomorphism $x^{a} \rightarrow$ $x^{a}+\xi^{a}$, corresponding to $L_{\text {sur }}$, will be given. This is important because it has not been done earlier, and therefore the properties of the current have not been explored. Here we will show that the corresponding charge $Q[\xi]$, calculated on the null surface for $\xi^{a}$ to be Killing, yields exactly one-quarter of the horizon area after multiplying it by $2 \pi / \kappa$, where $\kappa$ is the acceleration of the observer or the surface gravity in the case of a black hole. Next, a definition of the bracket among the charges will be given. This will be done by taking variation of the charge $Q\left[\xi_{1}\right]$ for another transformation $x^{a} \rightarrow x^{a}+$ $\xi_{2}^{a}$. Finally, we need to calculate all these quantities for a particular diffeomorphism. To identify the relevant diffeomorphisms from which the algebra has to be constructed, following our earlier work [55], we use the criterion that the diffeomorphism should leave the near horizon form of the metric invariant in some nonsingular coordinate system. This will lead to a set of diffeomorphism vectors for which the Fourier components of the bracket among the charges will be exactly similar to Virasoro algebra. It is then very easy to identify the zero mode eigenvalue and the central extension. Substitution of all these values in the Cardy formula [1012] will yield exactly the Bekenstein-Hawking entropy $[1,2]$. A similar analysis was done in [53] based on the Noether current corresponding to $L_{\text {bulk }}$ [64-66]. In this calculation, to obtain the correct value of the entropy, a particular boundary condition (Dirichlet or Neumann) was used. But its physical significance is not well understood.

Before going into the main calculation, let us summarize the main features of the present analysis.

(i) The first is the technical aspect. To obtain the correct entropy, in most of the earlier works, one had to either shift the zero mode eigenvalue [8] or choose a parameter contained in the Fourier modes of $\xi^{a}$ as the surface gravity $\kappa$ [53] or both [54]. Here we will show that none of the ad hoc prescriptions will be required.

(ii) The important one is the simplicity of the criterion (near horizon structure of the metric remains invariant in some nonsingular coordinate system) to find the relevant diffeomorphisms for which we obtain the Virasoro algebra. This was first introduced by us [55] in this context. The significance of this choice is that the full set of diffeomorphism symmetry of the theory is now reduced to a subset which respects the existence of horizon in a given coordinate system. Hence it may happen that some of the original gauge degrees of freedom (which could have been eliminated by certain diffeomorphisms which are now disallowed) now being effectively upgraded to physical degrees of freedom as far as a particular class of observers are concerned. So all the thermodynamic quantities, attributed to the horizon, become observer dependent. 
(iii) In the present analysis we will not need any use of boundary condition like Dirichlet or Neumann to obtain the exact form of the entropy.

(iv) Since our analysis will be completely based on $L_{\text {sur }}$, where no information about $L_{\text {bulk }}$ is needed, it will definitely illuminate the emergent paradigm of gravity, particularly the holographic aspects in the action.

We will discuss later more on different aspects and significance of our results.

The organization of the paper is as follows. In Section 2, the derivation of the Noether current for the $L_{\text {sur }}$ will be presented explicitly. Next we will give the definition of the bracket among the charges and the relevant diffeomorphisms based on the invariance of horizon structure criterion. Section 4 will be devoted to show that the Fourier mode of the bracket is exactly like the Virasoro algebra which by the Cardy formula will lead to Bekenstein-Hawking entropy. Finally, we will conclude.

\section{Derivation of Noether Current from the Surface Term of Einstein-Hilbert Action}

In this section, a detailed derivation of the Noether current and the potential corresponding to the surface term of $\mathrm{EH}$ action will be presented. Then we will calculate the charge on the Rindler horizon.

The Lagrangian corresponding to the surface term is given by [56]

$$
L_{\text {sur }}=\partial_{a}\left(\sqrt{-g} S^{a}\right)
$$

where

$$
S^{a}=2 Q_{c k}^{a d} g^{b k} \Gamma_{b d}^{c}, \quad Q_{c k}^{a d}=\frac{1}{2}\left(\delta_{c}^{a} \delta_{k}^{d}-\delta_{k}^{a} \delta_{c}^{d}\right) .
$$

Here the normalization $1 / 16 \pi G$ is omitted and it will be inserted where necessary. Now our task is to find the variations of both sides of (1) for a diffeomorphism $x^{\prime a}=x^{a}+\xi^{a}$ and then equate them. The variation we will consider here is the Lie variation which is defined, in general, as

$$
\delta A=A\left(x^{\prime}\right)-A^{\prime}\left(x^{\prime}\right),
$$

where $A\left(x^{\prime}\right)=A(x+\xi)=A(x)+\xi^{a} \partial_{a} A(x)$ and $A(x)$ and $A^{\prime}\left(x^{\prime}\right)$ are evaluated in two different coordinate systems $x$ and $x^{\prime}$, respectively. In the following, for the notational simplicity, we will denote $A(x)$ as $A$.

The variation of the right-hand side of (1) is given by

$$
\begin{aligned}
\delta L_{\text {sur }} & =\partial_{a}\left[\delta\left(\sqrt{-g} S^{a}\right)\right]=\partial_{a}\left[S^{a} \delta(\sqrt{-g})+\sqrt{-g} \delta S^{a}\right] \\
& =\partial_{a}\left[\frac{S^{a}}{2} \sqrt{-g} g^{b c} \delta g_{b c}+\sqrt{-g} \delta S^{a}\right] .
\end{aligned}
$$

Since $g_{a b}$ is a tensor, for the Lie variation, $\delta g_{a b}$ is expressed by the Lie derivative and is given by

$$
\delta g_{a b}=\nabla_{a} \xi_{b}+\nabla_{b} \xi_{a}
$$

Therefore,

$$
\delta L_{\text {sur }}=\partial_{a}\left[S^{a} \partial_{b}\left(\sqrt{-g} \xi^{b}\right)+\sqrt{-g} \delta S^{a}\right] .
$$

On the other hand, since $S^{a}$ is not a tensor, its variation cannot be expressed by simple Lie derivative. To find $\delta S^{a}$ we will use the general definition (3). Let us first calculate $S^{\prime a}\left(x^{\prime}\right)$. Under the change $x^{\prime a}=x^{a}+\xi^{a}$ we have

$$
\begin{aligned}
& \frac{\partial x^{\prime a}}{\partial x^{b}}=\delta_{b}^{a}+\partial_{b} \xi^{a} \\
& \frac{\partial x^{b}}{\partial x^{\prime a}}=\delta_{a}^{b}-\partial_{a} \xi^{b}
\end{aligned}
$$

Here we considered infinitesimal change, and so the terms from $\partial \xi \partial \xi$ have been ignored. This will be followed in later analysis. Hence,

$$
\begin{aligned}
\Gamma_{b c}^{\prime a}\left(x^{\prime}\right) & =\Gamma_{b c}^{a}-\Gamma_{b d}^{a} \partial_{c} \xi^{d}-\Gamma_{c d}^{a} \partial_{b} \xi^{d}+\Gamma_{b c}^{d} \partial_{d} \xi^{a}-\partial_{b} \partial_{c} \xi^{a} \\
g^{\prime b k}\left(x^{\prime}\right) & =g^{b k}+g^{b f} \partial_{f} \xi^{k}+g^{k f} \partial_{f} \xi^{b} \\
Q_{c k}^{\prime a d}\left(x^{\prime}\right) & =Q_{c k}^{a d}
\end{aligned}
$$

Substitution of these in $S^{\prime a}\left(x^{\prime}\right)=2 Q_{c k}^{\prime a d}\left(x^{\prime}\right) g^{\prime b k}\left(x^{\prime}\right) \Gamma_{b d}^{\prime c}\left(x^{\prime}\right)$ leads to

$$
S^{\prime a}\left(x^{\prime}\right)=S^{a}+S^{b} \partial_{b} \xi^{a}-g^{b d} \partial_{b} \partial_{d} \xi^{a}+g^{a b} \partial_{b} \partial_{c} \xi^{c} .
$$

Another one is given by

$$
S^{a}\left(x^{\prime}\right)=S^{a}\left(x^{b}+\xi^{b}\right)=S^{a}+\xi^{b} \partial_{b} S^{a} .
$$

Therefore, according to (3), the Lie variation of $S^{a}$ due to the diffeomorphism is

$$
\delta S^{a}=S^{a}\left(x^{\prime}\right)-S^{\prime a}\left(x^{\prime}\right)=\xi^{b} \partial_{b} S^{a}-S^{b} \partial_{b} \xi^{a}+M^{a},
$$

where

$$
M^{a}=g^{b d} \partial_{b} \partial_{d} \xi^{a}-g^{a b} \partial_{b} \partial_{c} \xi^{c}
$$

Substituting this in (6) we obtain the variation of right-hand side of (1) as

$$
\delta L_{\text {sur }}=\partial_{a}\left[\partial_{b}\left(\sqrt{-g} S^{a} \xi^{b}\right)-\sqrt{-g} S^{b} \partial_{b} \xi^{a}+\sqrt{-g} M^{a}\right] .
$$

Next we find the variation of left-hand side of (1), that is, $L_{\text {sur }}$. For this we will start from the following relation:

$$
L_{\text {sur }}=\sqrt{-g}\left(L_{g}-L_{\text {quad }}\right) \text {, }
$$

where

$$
L_{g}=R ; \quad L_{\text {quad }}=2 Q_{a}^{b c d} \Gamma_{d k}^{a} \Gamma_{b c}^{k}
$$


with $Q_{a}^{b c d}=(1 / 2)\left(\delta_{a}^{c} g^{b d}-\delta_{\mathrm{a}}^{d} g^{b c}\right)$. Since $L_{g}$ is a scalar, by the definition of Lie derivative, $\delta L_{g}=\xi^{a} \partial_{a} L_{g}$. Therefore using (5) we find

$$
\begin{aligned}
\delta L_{\text {sur }}= & \delta\left(\sqrt{-g} L_{g}\right)-\delta\left(\sqrt{-g} L_{\text {quad }}\right) \\
= & \partial_{a}\left(\sqrt{-g} \xi^{a} L_{g}\right)-\partial_{a}\left(\sqrt{-g} \xi^{a}\right) L_{\text {quad }}-\sqrt{-g} \delta L_{\text {quad }} \\
= & \partial_{a}\left[\sqrt{-g} \xi^{a}\left(L_{g}-L_{\text {quad }}\right)\right]+\sqrt{-g} \xi^{a} \partial_{a} L_{\text {quad }} \\
& -\sqrt{-g} \delta L_{\text {quad }} \\
= & \partial_{a}\left(\xi^{a} L_{\text {sur }}\right)+\sqrt{-g} \xi^{a} \partial_{a} L_{\text {quad }}-\sqrt{-g} \delta L_{\text {quad }} .
\end{aligned}
$$

To find $\delta L_{\text {quad }}$, we will proceed as earlier. Under the change $x^{\prime a}=x^{a}+\xi^{a}, L_{\text {quad }}^{\prime}\left(x^{\prime}\right)$ is calculated as

$$
\begin{aligned}
& L_{\text {quad }}^{\prime}\left(x^{\prime}\right) \\
& =2 Q_{a}^{\prime b c d}\left(x^{\prime}\right) \Gamma_{d k}^{\prime a}\left(x^{\prime}\right) \Gamma_{b c}^{\prime k}\left(x^{\prime}\right) \\
& =L_{\text {quad }}+g^{b c} \Gamma_{b c}^{k} \partial_{d} \partial_{k} \xi^{d}+g^{b c} \Gamma_{d k}^{d} \partial_{b} \partial_{c} \xi^{k}-g^{b d} \Gamma_{d k}^{c} \partial_{b} \partial_{c} \xi^{k},
\end{aligned}
$$

where (8) has been used. This can be expressed in terms of $M^{a}$ in the following way. The second term on the right-hand side can be expressed in the following form:

$$
\begin{aligned}
\sqrt{-g} g^{b c} \Gamma_{b c}^{k} \partial_{d} \partial_{k} \xi^{d} \\
\quad=\left[\sqrt{-g} g^{b c} g^{a k} \partial_{b} g_{a c}-g^{a k} \partial_{a}(\sqrt{-g})\right] \partial_{d} \partial_{k} \xi^{d} \\
\quad=-\partial_{a}\left(\sqrt{-g} g^{a k}\right) \partial_{d} \partial_{k} \xi^{d},
\end{aligned}
$$

where in the above we used $g^{b c} g^{a k} \partial_{b} g_{a c}=-\partial_{a} g^{a k}$. The third term of (17) reduces to

$$
\sqrt{-g} g^{b c} \Gamma_{d k}^{d} \partial_{b} \partial_{c} \xi^{k}=\partial_{k}(\sqrt{-g}) g^{b c} \partial_{b} \partial_{c} \xi^{k} .
$$

Similarly, the last term can be expressed as

$$
\begin{aligned}
2 \sqrt{-g} g^{b d} \Gamma_{d k}^{c} \partial_{b} \partial_{c} \xi^{k} & =\sqrt{-g} g^{b d} g^{c a} \partial_{k} g_{a d} \partial_{b} \partial_{c} \xi^{k} \\
& =-\sqrt{-g} \partial_{k}\left(g^{b c}\right) \partial_{b} \partial_{c} \xi^{k}
\end{aligned}
$$

where in the last line $g^{b d} g^{c a} \partial_{k} g_{a d}=-\partial_{k} g^{b c}$ has been used. Substituting all these in (17) we obtain

$$
\begin{aligned}
& L_{\text {quad }}^{\prime}\left(x^{\prime}\right) \\
& =L_{\text {quad }}-\frac{1}{\sqrt{-g}}\left[\partial_{a}\left(\sqrt{-g} g^{a k}\right) \partial_{d} \partial_{k} \xi^{d}+\partial_{k}\left(\sqrt{-g} g^{b c}\right) \partial_{b} \partial_{c} \xi^{k}\right] \\
& =L_{\text {quad }}+\frac{1}{\sqrt{-g}} \partial_{a}\left(\sqrt{-g} M^{a}\right) .
\end{aligned}
$$

On the other hand,

$$
L_{\text {quad }}\left(x^{\prime}\right)=L_{\text {quad }}\left(x^{a}+\xi^{a}\right)=L_{\text {quad }}+\xi^{a} \partial_{a} L_{\text {quad }} \cdot
$$

Hence,

$$
\begin{aligned}
\sqrt{-g} \delta L_{\text {quad }} & =\sqrt{-g} L_{\text {quad }}\left(x^{\prime}\right)-\sqrt{-g} L_{\text {quad }}^{\prime}\left(x^{\prime}\right) \\
& =\sqrt{-g} \xi^{a} \partial_{a} L_{\text {quad }}-\partial_{a}\left(\sqrt{-g} M^{a}\right) .
\end{aligned}
$$

Substituting this in (16) we obtain

$$
\delta L_{\text {sur }}=\partial_{a}\left(\xi^{a} L_{\text {sur }}+\sqrt{-g} M^{a}\right)
$$

Now equating (13) and (24) we obtain $\partial_{a} J^{a}[\xi]=0$, where the conserved Noether current $J^{a}[\xi]$ is given by

$$
J^{a}[\xi]=-\partial_{b}\left(\sqrt{-g} S^{a} \xi^{b}\right)+\sqrt{-g} S^{b} \partial_{b} \xi^{a}+\xi^{a} L_{\text {sur }}
$$

Finally, using $L_{\text {sur }}=\partial_{a}\left(\sqrt{-g} S^{a}\right)$ in the above, we can express the current as the divergence of an antisymmetric two-index quantity:

$$
J^{a}[\xi]=\partial_{b}\left[\sqrt{-g}\left(\xi^{a} S^{b}-\xi^{b} S^{a}\right)\right]=\partial_{b}\left[\sqrt{-g} J^{a b}[\xi]\right] .
$$

It is evident that the anti-symmetric object $J^{a b}[\xi]$ is not a tensor and it is usually called the Noether potential. Therefore, inserting the proper normalization, the charge is given by

$$
Q[\xi]=\frac{1}{32 \pi G} \int_{\mathscr{H}} d \Sigma_{a b} \sqrt{h} J^{a b}[\xi]
$$

where $d \Sigma_{a b}=-d^{2} x\left(N_{a} M_{b}-N_{b} M_{a}\right)$ is the surface element of the 2-dimensional surface $\mathscr{H}$ and $h$ is the determinant of the corresponding metric. Since our present discussion will be near the horizon, we choose the unit normals $N_{a}$ and $M_{a}$ as spacelike and timelike, respectively.

Now we will calculate charge (27) explicitly on the horizon. This will be done by considering the form of the metric near the horizon:

$$
d s^{2}=-2 \kappa x d t^{2}+\frac{1}{2 \kappa x} d x^{2}+d x_{\perp}^{2},
$$

where $x_{\perp}$ represents the transverse coordinates. The metric has a timelike Killing vector $\chi^{a}=(1,0,0,0)$ and the Killing horizon is given by $\chi^{2}=0$; that is, $x=0$. The nonzero Christoffel connections are

$$
\Gamma_{t x}^{t}=\frac{1}{2 x}, \quad \Gamma_{t t}^{x}=2 \kappa^{2} x, \quad \Gamma_{x x}^{x}=-\frac{1}{2 x} .
$$

For metric (28) we find

$$
N^{a}=(0, \sqrt{2 \kappa x}, 0,0), \quad M^{a}=\left(\frac{1}{\sqrt{2 \kappa x}}, 0,0,0\right),
$$

and hence $d \Sigma_{t x}=-d^{2} x$. Also, (2) yields

$$
S^{t}=0, \quad S^{x}=-2 \kappa .
$$


Therefore,

$$
J^{t x}=\left(\xi^{t} S^{x}-\xi^{x} S^{t}\right)=-2 \kappa \xi^{t}
$$

Now if $\xi^{a}$ is a Killing vector, then $\xi^{t}=\chi^{t}=1$, and so calculating charge (27) explicitly we find

$$
Q[\xi=\chi]=\frac{\kappa A_{\perp}}{8 \pi G}
$$

where $A_{\perp}=\int_{\mathscr{C}} d^{2} x$ is the horizon cross-section area. Multiplying it by the periodicity of time coordinate $2 \pi / \kappa$ we obtain exactly the entropy: one-quarter of horizon area. Moreover, the above can be expressed as $Q[\xi=\chi]=T S$, where $T=\kappa / 2 \pi$ is the temperature of the horizon and $S=A_{\perp / 4 G}$ is the entropy. Therefore one can call it the Noether energy. Such interpretaion was done earlier in $[67,68]$.

So far we found that the Noether charge corresponding to the surface term of EH action alone led to the entropy of the Rindler horizon. This was shown earlier for the charge coming from the total EH action [61-63]. Therefore, the present analysis revealed that it may be possible that the information is actually encoded in the surface term rather than the bulk term. Then the natural question arises: what are the degrees of freedom responsible for this entropy? So far they are not known. In the next couple of sections we will give an idea on the nature of the possible degrees of freedom in the context of Virasoro algebra and Cardy formula.

\section{Bracket among the Charges and the Diffeomorphism Generators}

In the previous section, we have given the expression for the charge (see (27)) for an arbitrary diffeomorphism. Here we will define the bracket among the charges. The relevant diffeomorphisms will be chosen by imposing a minimum condition on the spacetime metric. The charge and the bracket will be then expressed in terms of these generators.

We will find the bracket following our earlier works [54, 55]. For this let us first calculate the following:

$$
\begin{aligned}
\delta_{\xi_{1}} & \left(\sqrt{-g} J^{a b}\left[\xi_{2}\right]\right) \\
= & \delta_{\xi_{1}}(\sqrt{-g}) J^{a b}\left[\xi_{2}\right]+\sqrt{-g} \delta_{\xi_{1}}\left(J^{a b}\left[\xi_{2}\right]\right) \\
= & -\frac{1}{2} \sqrt{-g} g_{m n} \delta_{\xi_{1}} g^{m n} J^{a b}\left[\xi_{2}\right] \\
& +\sqrt{-g}\left[\left(\delta_{\xi_{1}} \xi_{2}^{a}\right) S^{b}+\xi_{2}^{a}\left(\delta_{\xi_{1}} S^{b}\right)-(a \longleftrightarrow b)\right] .
\end{aligned}
$$

Using

$$
\begin{aligned}
\delta_{\xi} g^{a b} & =\mathfrak{E}_{\xi} g^{a b}=-\nabla^{a} \xi^{b}-\nabla^{b} \xi^{a}, \\
\delta_{\xi} \Gamma_{b c}^{a} & =\nabla_{b} \nabla_{c} \xi^{a}+R_{c m b}^{a} \xi^{m},
\end{aligned}
$$

and in addition the expression for $S^{a}$, given by (2), we obtain

$$
\begin{aligned}
& \delta_{\xi_{1}}\left(\sqrt{-g} J^{a b}\left[\xi_{2}\right]\right) \\
& =\sqrt{-g}\left[\nabla_{m} \xi_{1}^{m} J^{a b}\left[\xi_{2}\right]\right. \\
& +\left\{\left(\xi_{1}^{m} \nabla_{m} \xi_{2}^{a}-\xi_{2}^{m} \nabla_{m} \xi_{1}^{a}\right) S^{b}\right. \\
& \quad+\xi_{2}^{a}\left(-2 \Gamma_{m n}^{b} \nabla^{m} \xi_{1}^{n}+\nabla_{m} \nabla^{m} \xi_{1}^{b}+2 R_{m}^{b} \xi_{1}^{m}\right. \\
& \left.\quad-\Gamma_{n m}^{n}\left(\nabla^{b} \xi_{1}^{m}+\nabla^{m} \xi_{1}^{b}\right)-\nabla_{m} \nabla^{b} \xi_{1}^{m}\right) \\
& -(a \longleftrightarrow b)\}] .
\end{aligned}
$$

For the present metric (28), $g=-1, R_{b}^{a}=0$, and hence $\sqrt{-g} \Gamma_{n m}^{n}=\partial_{m}(\sqrt{-} g)=0$. Therefore

$$
\begin{aligned}
& \delta_{\xi_{1}}\left(\sqrt{-g} J^{a b}\left[\xi_{2}\right]\right) \\
& =\left[\nabla_{m} \xi_{1}^{m} J^{a b}\left[\xi_{2}\right]\right. \\
& \quad+\frac{1}{16 \pi G}\left\{\left(\xi_{1}^{m} \nabla_{m} \xi_{2}^{a}-\xi_{2}^{m} \nabla_{m} \xi_{1}^{a}\right) S^{b}\right. \\
& \quad+\xi_{2}^{a}\left(-2 \Gamma_{m n}^{b} \nabla^{m} \xi_{1}^{n}+\nabla_{m} \nabla^{m} \xi_{1}^{b}-\nabla_{m} \nabla^{b} \xi_{1}^{m}\right) \\
& \quad-(a \longleftrightarrow b)\}] \\
& \equiv K_{12}^{a b} \quad
\end{aligned}
$$

Finally we define a bracket as

$$
\left[Q\left[\xi_{1}\right], Q\left[\xi_{2}\right]\right]:=\frac{1}{2} \int_{\mathscr{H}} d \Sigma_{a b} \sqrt{h}\left[K_{12}^{a b}-(1 \longleftrightarrow 2)\right],
$$

which for the present metric (28) reduces to

$$
\left[Q\left[\xi_{1}\right], Q\left[\xi_{2}\right]\right]:=-\int_{\mathscr{C}} d^{2} x\left[K_{12}^{t x}-(1 \longleftrightarrow 2)\right] .
$$

To calculate the above bracket we need to know about the generators $\xi^{a}$. We will determine them by using the condition that the horizon structure remains invariant in some nonsingular coordinate system. For that let us first express metric (28) in Gaussian null (or Bondi like) coordinates:

$$
d u=d t-\frac{d x}{2 \kappa x}, \quad d X=d x .
$$

In these coordinates the metric reduces to the following form:

$$
d s^{2}=-2 \kappa X d u^{2}-2 d u d X+d x_{\perp}^{2} .
$$

Now impose the condition that the metric coefficients $g_{X X}$ and $g_{u X}$ do not change under the diffeomorphism; that is,

$$
\mathfrak{E}_{\tilde{\xi}} g_{X X}=0, \quad \mathfrak{E}_{\tilde{\xi}} g_{u X}=0,
$$


where $\mathfrak{E}_{\tilde{\xi}}$ is the Lie derivative along the vector $\tilde{\xi}$. These lead to

$$
\begin{aligned}
& \mathfrak{E}_{\tilde{\xi}} g_{X X}=-2 \partial_{X} \widetilde{\xi}^{u}=0, \\
& \mathfrak{E}_{\tilde{\xi}} g_{u X}=-\partial_{u} \widetilde{\xi}^{u}-2 \kappa X \partial_{X} \widetilde{\xi}^{u}-\partial_{X} \tilde{\xi}^{X}=0 .
\end{aligned}
$$

The solutions are

$$
\begin{aligned}
\tilde{\xi}^{u} & =F\left(u, x_{\perp}\right), \\
\tilde{\xi}^{X} & =-X \partial_{u} F\left(u, x_{\perp}\right) .
\end{aligned}
$$

The condition $\mathfrak{E}_{\tilde{\xi}} g_{u u}=0$ is automatically satisfied near the horizon, because use of the above solutions leads to $\mathfrak{E}_{\tilde{\xi}} g_{u u}=$ $\mathcal{O}(X)$. These conditions appeared earlier in [69] in the context of late time symmetry near the black hole horizon. Finally expressing (44) in the old coordinates $(t, x)$ we find

$$
\xi^{t}=T-\frac{1}{2 \kappa} \partial_{t} T, \quad \xi^{x}=-x \partial_{t} T,
$$

where $T\left(t, x, x_{\perp}\right)=F\left(u, x_{\perp}\right)$.

Next we calculate $K_{12}^{t x}$ from (37) for the present case. Since $S^{t}=0$, we find

$$
\begin{aligned}
K_{12}^{t x}= & \nabla_{m} \xi_{1}^{m} \xi_{2}^{t} S^{x}+\left(\xi_{1}^{m} \nabla_{m} \xi_{2}^{t}-\xi_{2}^{m} \nabla_{m} \xi_{1}^{t}\right) S^{x} \\
& +\xi_{2}^{t}\left(-2 \Gamma_{m n}^{x} \nabla^{m} \xi_{1}^{n}+\nabla_{m} \nabla^{m} \xi_{1}^{x}-\nabla_{m} \nabla^{x} \xi_{1}^{m}\right) \\
& -\xi_{2}^{x}\left(-2 \Gamma_{m n}^{t} \nabla^{m} \xi_{1}^{n}+\nabla_{m} \nabla^{m} \xi_{1}^{t}-\nabla_{m} \nabla^{t} \xi_{1}^{m}\right) .
\end{aligned}
$$

Now since the integration (39) will ultimately be evaluated on the horizon, we will find the value of each term of the above very near to the horizon. Therefore, using (29), (31), and the form of the generators (45) we obtain the values of each term of the above expression near the horizon $x=0$ as

$$
\begin{gathered}
\nabla_{m} \xi_{1}^{m} \xi_{2}^{t} S^{x}=T_{2} \partial_{t}^{2} T_{1}-\frac{1}{2 \kappa} \partial_{t}^{2} T_{1} \partial_{t} T_{2} \\
\xi_{1}^{m} \nabla_{m} \xi_{2}^{t} S^{x}=-\kappa T_{1} \partial_{t} T_{2}+\kappa T_{2} \partial_{t} T_{1}+T_{1} \partial_{t}^{2} T_{2}-\frac{1}{2 \kappa} \partial_{t} T_{1} \partial_{t}^{2} T_{2} \\
-\xi_{2}^{m} \nabla_{m} \xi_{1}^{t} S^{x}=\kappa T_{2} \partial_{t} T_{1}-\kappa T_{1} \partial_{t} T_{2}-T_{2} \partial_{t}^{2} T_{1}+\frac{1}{2 \kappa} \partial_{t} T_{2} \partial_{t}^{2} T_{1} \\
-2 \xi_{2}^{t} \Gamma_{m n}^{x} \nabla^{m} \xi_{1}^{n}=-T_{2} \partial_{t}^{2} T_{1}+\frac{1}{2 \kappa} \partial^{2} T_{1} \partial_{t} T_{2} \\
\xi_{2}^{t} \nabla_{m} \nabla^{m} \xi_{1}^{x}=\frac{1}{2 \kappa} T_{2} \partial_{t}^{3} T_{1}-\frac{1}{4 \kappa^{2}} \partial_{t}^{3} T_{1} \partial_{t} T_{2}-2 \kappa T_{2} \partial_{t} T_{1} \\
+\partial_{t} T_{1} \partial_{t} T_{2}+T_{2} \partial_{t}^{2} T_{1}-\frac{1}{2 \kappa} \partial_{t}^{2} T_{1} \partial_{t} T_{2} \\
-\xi_{2}^{t} \nabla_{m} \nabla^{x} \xi_{1}^{m}=0 \\
2 \xi_{2}^{x} \Gamma_{m n}^{t} \nabla^{m} \xi_{1}^{n}=-\frac{1}{2 \kappa} \partial_{t}^{2} T_{1} \partial_{t} T_{2}-2 \kappa x \partial_{x} T_{1} \partial_{t} T_{2} \\
-\xi_{2}^{x} \nabla_{m} \nabla^{m} \xi_{1}^{t}=\frac{1}{4 \kappa^{2}} \partial_{t}^{3} T_{1} \partial_{t} T_{2} \\
\xi_{2}^{x} \nabla_{m} \nabla^{t} \xi_{1}^{m}=-\frac{1}{4 \kappa^{2}} \partial_{t}^{3} T_{1} \partial_{t} T_{2} .
\end{gathered}
$$

So near the horizon (46) reduces to

$$
\begin{aligned}
K_{12}^{t x}= & -2 \kappa T_{1} \partial_{t} T_{2}+T_{1} \partial_{t}^{2} T_{2}-\frac{1}{2 \kappa}\left(\partial_{t} T_{1} \partial_{t}^{2} T_{2}+\partial_{t}^{2} T_{1} \partial_{t} T_{2}\right) \\
& +\frac{1}{2 \kappa} T_{2} \partial_{t}^{3} T_{1}+\partial_{t} T_{1} \partial_{t} T_{2}-\frac{1}{4 \kappa^{2}} \partial^{3} T_{1} \partial_{t} T_{2} .
\end{aligned}
$$

Substituting this in (39) and inserting the normalization factor, we obtain the expression for the bracket

$$
\begin{aligned}
& {\left[Q\left[\xi_{1}\right], Q\left[\xi_{2}\right]\right]} \\
& :=\frac{1}{16 \pi G} \int_{\mathscr{H}} d^{2} x\left[2 \kappa\left(T_{1} \partial_{t} T_{2}-T_{2} \partial_{t} T_{1}\right)\right. \\
& -\left(T_{1} \partial_{t}^{2} T_{2}-T_{2} \partial_{t}^{2} T_{1}\right) \\
& +\frac{1}{2 \kappa}\left(T_{1} \partial_{t}^{3} T_{2}-T_{2} \partial_{t}^{3} T_{1}\right) \\
& \left.+\frac{1}{4 \kappa^{2}}\left(\partial_{t}^{3} T_{1} \partial_{t} T_{2}-\partial_{t}^{3} T_{2} \partial_{t} T_{1}\right)\right] .
\end{aligned}
$$

Similarly, (27) yields

$$
Q[\xi]=\frac{1}{8 \pi G} \int d^{2} x\left(\kappa T-\frac{1}{2} \partial_{t} T\right)
$$

A couple of comments are in order. It must be noted that, in finding the expression for bracket (49), no use of boundary conditions (Dirichlet or Neumann) has been used. Earlier this was used for the case of $L_{\text {bulk }}$ to throw away the noncovariant terms in the bracket without giving any physical meaning [53]. Also, we did not use the condition $\delta_{\xi_{1}} \xi_{2}^{a}=0$ (see (34)) which was adopted in earlier works. For instance, see $[8,53]$. This is logically correct since $\delta_{\xi_{1}} \xi_{2}^{a}=0$ contradicts the algebra among the Fourier modes of the diffeomorphisms (see (52), in next section).

\section{Virasoro Algebra and Entropy}

In this section, the Fourier modes of the bracket and the charge will be found out. We will show that for a particular ansatz for the Fourier modes of the generators will lead to the Virasoro algebra. Finally using the Cardy formula, the entropy will be calculated.

Consider the Fourier decompositions of $T_{1}$ and $T_{2}$ :

$$
T_{1}=\sum_{m} A_{m} T_{m}, \quad T_{2}=\sum_{n} B_{n} T_{n}
$$

where $A_{m}^{*}=A_{-m}$ and $B_{n}^{*}=B_{-n}$. The Fourier modes $T_{m}$ will be chosen such that the Fourier modes of the diffeomorphisms (44) obey one subalgebra isomorphic to Diff. $S^{1}$ :

$$
i\left\{\xi_{m}, \xi_{n}\right\}^{a}=(m-n) \xi_{m+n}^{a}
$$


where $\{$,$\} is the Lie bracket. Now with the use of (51), let us$ first find the Fourier modes of bracket (49) and charge (50). Substitution of (51) in (49) yields

$$
\begin{aligned}
& {\left[Q\left[\xi_{1}\right], Q\left[\xi_{2}\right]\right]} \\
& :=\sum_{m, n} \frac{C_{m, n}}{16 \pi G} \int_{\mathscr{H}} d^{2} x\left[2 \kappa\left(T_{m} \partial_{t} T_{n}-T_{n} \partial_{t} T_{m}\right)\right. \\
& -\left(T_{m} \partial_{t}^{2} T_{n}-T_{n} \partial_{t}^{2} T_{m}\right) \\
& +\frac{1}{2 \kappa}\left(T_{m} \partial_{t}^{3} T_{n}-T_{n} \partial_{t}^{3} T_{m}\right) \\
& \left.+\frac{1}{4 \kappa^{2}}\left(\partial_{t}^{3} T_{m} \partial_{t} T_{n}-\partial_{t}^{3} T_{n} \partial_{t} T_{m}\right)\right],
\end{aligned}
$$

where $C_{m, n}=A_{m} B_{n}$ and so $C_{m, n}^{*}=C_{-m,-n}$. Next defining the Fourier modes of $\left[Q\left[\xi_{1}\right], Q\left[\xi_{2}\right]\right]$ as

$$
\left[Q\left[\xi_{1}\right], Q\left[\xi_{2}\right]\right]=\sum_{m, n} C_{m, n}\left[Q_{m}, Q_{n}\right]
$$

we find

$$
\begin{aligned}
& {\left[Q_{m}, Q_{n}\right]} \\
& \qquad=\frac{1}{16 \pi G} \int_{\mathscr{H}} d^{2} x\left[2 \kappa\left(T_{m} \partial_{t} T_{n}-T_{n} \partial_{t} T_{m}\right)\right. \\
& -\left(T_{m} \partial_{t}^{2} T_{n}-T_{n} \partial_{t}^{2} T_{m}\right) \\
& +\frac{1}{2 \kappa}\left(T_{m} \partial_{t}^{3} T_{n}-T_{n} \partial_{t}^{3} T_{m}\right) \\
& \left.+\frac{1}{4 \kappa^{2}}\left(\partial_{t}^{3} T_{m} \partial_{t} T_{n}-\partial_{t}^{3} T_{n} \partial_{t} T_{m}\right)\right]
\end{aligned}
$$

Similarly from (50), the Fourier modes of the charge are given by

$$
Q_{m}=\frac{1}{8 \pi G} \int d^{2} x\left(\kappa T_{m}-\frac{1}{2} \partial_{t} T_{m}\right),
$$

where $Q[\xi]=\sum_{m} A_{m} Q_{m}$. It must be noted that the present expression (56) is exactly identical to that obtained in [55] for the York-Gibbons-Hawking surface term, whereas the other expression (55) is different by a total derivative term. This may be because these two surface terms are not exactly the same. But we will show that final result for the bracket is identical to the earlier analysis, because the total derivative term will not contribute to an ansatz for $T_{m}$.

To calculate the previous expressions (55) and (56) explicitly we need to have $T_{m}$ 's. Following the earlier arguments, we choose

$$
T_{m}=\frac{1}{\alpha} e^{i m\left(\alpha t+g(x)+p \cdot x_{\perp}\right)}
$$

such that they satisfy algebra (52). Here $\alpha$ is a constant, $p$ is an integer, and $g(x)$ is a function which is regular at the horizon. This is a standard choice in these computations and has been used several times in the literature $[7,8,53]$. It must be noted that the transverse directions are noncompact due to our Rindler approximations and so we will assume that $T_{m}$ is periodic in the transverse coordinates with the periodicities $L_{y}$ and $L_{z}$ on $y$ and $z$, respectively. Now substituting (57) in (55) and (56) and then integrating over the cross-sectional area $A_{\perp}=L_{y} L_{z}$, we obtain

$$
\begin{gathered}
Q_{m}=\frac{A_{\perp}}{8 \pi G} \frac{\kappa}{\alpha} \delta_{m, 0}, \\
i\left[Q_{m}, Q_{n}\right]:=\frac{A_{\perp}}{8 \pi G} \frac{\kappa}{\alpha}(m-n) \delta_{m+n, 0}+n^{3} \frac{A_{\perp}}{16 \pi G} \frac{\alpha}{\kappa} \delta_{m+n, 0} .
\end{gathered}
$$

Using (58), (59) can be reexpressed as

$$
i\left[Q_{m}, Q_{n}\right]:=(m-n) Q_{m+n}+n^{3} \frac{A_{\perp}}{16 \pi G} \frac{\alpha}{\kappa} \delta_{m+n, 0} .
$$

This is exactly identical to Virasoro algebra with the central charge $C$ being identified as

$$
\frac{C}{12}=\frac{A_{\perp}}{16 \pi G} \frac{\alpha}{\kappa} .
$$

The zero mode eigenvalue is evaluated from (58) for $m=0$ :

$$
Q_{0}=\frac{A_{\perp}}{8 \pi G} \frac{\kappa}{\alpha} .
$$

Finally using the Cardy formula [10-12], we obtain the entropy as

$$
S=2 \pi \sqrt{\frac{C Q_{0}}{6}}=\frac{A_{\perp}}{4 G},
$$

which is exactly the Bekenstein-Hawking entropy.

\section{Conclusions}

It has already been observed that several interesting features and pieces of information can be obtained from the surface term without incorporating the bulk term of the gravity action. In this paper we studied the surface term of the Einstein-Hilbert (EH) action in the context of Noether current. So far we know that this has not been attempted before. First the current was derived for an arbitrary diffeomorphism by using Noether prescription. Then we showed that the charge evaluated on the horizon for a Killing vector led to the Bekenstein-Hawking entropy after multiplying it by $2 \pi / \kappa$. But till now, it is not known about the degrees of freedom responsible for the entropy. Here we addressed the issue and tried to shed some light. This has been discussed in the context of Virasoro algebra and Cardy formula.

In this paper, we defined the bracket among the charges. It was done, in the sprite of our earlier works $[54,55]$, by taking the variation of the Noether potential $J^{a b}\left[\xi_{1}\right]$ for a different diffeomorphism $x^{a} \rightarrow x^{a}+\xi_{2}^{a}$ and then an anti-symmetric combination between the indices 1 and 2 and integrating over the horizon surface. To achieve the final form, we did not use Einstein's equation of motion or any ambiguous prescription, like vanishing of the variation of diffeomorphism parameter 
$\xi^{a}$, certain boundary conditions (e.g., Dirichlet or Neumann), and so forth. For explicit evaluation of our bracket, the spacetime was considered as the Rindler metric. The relevant diffeomorphisms were identified by using a very simple, physically motivated condition: the diffeomorphisms keep the horizon structure of the metric invariant in some nonsingular coordinate system. It turned out that the Fourier modes of the bracket are similar to the Virasoro algebra. Identifying the central charge and the zero mode eigenvalue and then using these in Cardy formula, we obtained exactly the BekensteinHawking entropy.

Let us now discuss in details what we have achieved in this paper. We first tabulate a couple of technical points.

(i) To obtain the exact expression for entropy we did not need any hand waving prescriptions, like shifting of the value of the zero mode eigenvalue or the specific choice of the value of the parameter $\alpha$ that appeared in Fourier modes of $T$ or both.

(ii) The relevant diffeomorphisms for invariance of the horizon structure can be obtained by various ways. Here our idea was to impose minimum constraints so that the bracket led to Virasoro algebra. It is also possible to have other choices of constraints to find the vectors $\xi^{a}$. For instance, the whole metric is invariant, and the diffeomorphisms come out to be the Killing vectors which in general do not exist for a general spacetime.

Finally, we discuss several conceptual aspects. The analysis presents a nice connection between the horizon entropy and the degrees of freedom which are responsible for it. In the usual cases, one always find that the concepts of degrees of freedom and entropy are absolute. These do not have any observer-dependent description. But in the case of gravity, as we know, the notion of temperature and entropy is observer dependent, and hence one can expect that the degrees of freedom may not be absolute. Here we showed that a certain class of observers which can see the horizon and keep the horizon structure invariant always attribute entropy. This signifies the fact that, among all the diffeomorphisms, some of them upgraded to real degrees of freedom which were originally gauge degrees of freedom and they have observerdependent notion. Also, everything we achieved here was done from surface term. This again illustrates the holographic nature of the gravity actions-either the bulk and the surface terms may duplicate the same information or the surface term alone contains all the information about the theory of gravity. Moreover, the methodology is general enough to discuss other theories of gravity.

\section{Acknowledgment}

The author thanks T. Padmanabhan for several useful discussions.

\section{References}

[1] J. D. Bekenstein, "Black holes and entropy," Physical Review D, vol. 7, no. 8, pp. 2333-2346, 1973.
[2] S. W. Hawking, "Black hole explosions?" Nature, vol. 248, no. 5443, pp. 30-31, 1974.

[3] T. Padmanabhan, "Thermodynamical aspects of gravity: new insights," Reports on Progress in Physics, vol. 73, Article ID 046901, 2010.

[4] T. Padmanabhan, "Lessons from classical gravity about the quantum structure of spacetime," Journal of Physics: Conference Series, vol. 306, Article ID 012001, 2011.

[5] R. Banerjee and B. R. Majhi, "Statistical origin of gravity," Physical Review D, vol. 81, Article ID 124006, 2010.

[6] B. R. Majhi, "Emergent gravity: from statistical point of view," Journal of Physics: Conference Series, vol. 405, Article ID 012020, 2012.

[7] S. Carlip, "Black hole entropy from conformai field theory in any dimension," Physical Review Letters, vol. 82, no. 14, pp. 2828-2831, 1999.

[8] S. Carlip, "Entropy from conformal field theory at killing horizons," Classical and Quantum Gravity, vol. 16, no. 10, pp. 33273348, 1999.

[9] J. D. Brown and M. Henneaux, "Central charges in the canonical realization of asymptotic symmetries: an example from three dimensional gravity," Communications in Mathematical Physics, vol. 104, no. 2, pp. 207-226, 1986.

[10] J. L. Cardy, "Operator content of two-dimensional conformally invariant theories," Nuclear Physics B, vol. 270, no. 2, pp. 186204, 1986.

[11] H. W. J. Blöte, J. L. Cardy, and M. P. Nightingale, "Conformal invariance, the central charge, and universal finite-size amplitudes at criticality," Physical Review Letters, vol. 56, no. 7, pp. 742-745, 1986.

[12] S. Carlip, "What we don't know about BTZ black hole entropy," Classical and Quantum Gravity, vol. 15, no. 11, pp. 3609-3625, 1998.

[13] S. N. Solodukhin, "Conformal description of horizon's states," Physics Letters B, vol. 454, no. 3-4, pp. 213-222, 1999.

[14] R. Brustein, "Causal boundary entropy from horizon conformal field theory," Physical Review Letters, vol. 86, no. 4, pp. 576-579, 2001.

[15] G. A. S. Dias and J. P. S. Lemos, "Conformal entropy from horizon states: Solodukhin's method for spherical, toroidal, and hyperbolic black holes in $D$-dimensional anti-de Sitter spacetimes," Physical Review D, vol. 74, no. 4, Article ID 044024, 2006.

[16] R. Banerjee, S. Gangopadhyay, and S. Kulkarni, "Hawking radiation and near horizon universality of chiral Virasoro algebra," General Relativity and Gravitation, vol. 42, no. 12, pp. 2865-2871, 2010.

[17] B. R. Majhi, "Gravitational anomalies and entropy," General Relativity and Gravitation, vol. 45, no. 2, pp. 345-357, 2013.

[18] F.-L. Lin and Y.-S. Wu, "Near-horizon Virasoro symmetry and the entropy of de Sitter space in any dimension," Physics Letters $B$, vol. 453, no. 3-4, pp. 222-228, 1999.

[19] V. O. Soloviev, "Black hole entropy from Poisson brackets: demystification of some calculations," Physical Review D, vol. 61, no. 2, Article ID 027502, 2000.

[20] M. Natsuume, T. Okamura, and M. Sato, "Three-dimensional gravity with a conformal scalar field and asymptotic Virasoro algebra," Physical Review D, vol. 61, no. 10, Article ID 104005, 2000.

[21] D. J. Navarro, J. Navarro-Salas, and P. Navarro, "Holography, degenerate horizons and entropy," Nuclear Physics B, vol. 580, no. 1-2, pp. 311-330, 2000. 
[22] J. Jing and M.-L. Yan, "Entropies of rotating charged black holes from conformal field theory at Killing horizons," Physical Review D, vol. 62, no. 10, Article ID 104013, 2000.

[23] S. Das, A. Ghosh, and P. Mitra, "Statistical entropy of Schwarzschild black strings and black holes," Physical Review D, vol. 63, no. 2, Article ID 024023, 2001.

[24] J. Jing and M.-L. Yan, "Statistical entropy of a stationary dilaton black hole from the Cardy formula," Physical Review D, vol. 63, no. 2, Article ID 024003, 2001.

[25] H. Terashima, "The Brown-Henneaux's central charge from the path-integral boundary condition," Physics Letters B, vol. 499, no. 1-2, pp. 229-232, 2001.

[26] M. Hotta, K. Sasaki, and T. Sasaki, "Diffeomorphism on the horizon as an asymptotic isometry of the Schwarzschild black hole," Classical and Quantum Gravity, vol. 18, no. 10, pp. 18231834, 2001.

[27] H. Terashima, "Path integral derivation of the Brown-Henneaux central charge," Physical Review D, vol. 64, no. 6, Article ID 064016, 2001.

[28] J.-i. Koga, "Asymptotic symmetries on Killing horizons," Physical Review D, vol. 64, no. 12, Article ID 124012, 2001.

[29] M.-I. Park, "The Hamiltonian dynamics of bounded spacetime and black hole entropy: the canonical method," Nuclear Physics $B$, vol. 634, no. 1-2, pp. 339-369, 2002.

[30] S. Carlip, "Near-horizon conformal symmetry and black hole entropy," Physical Review Letters, vol. 88, no. 24, Article ID 241301, 2002.

[31] M. Cvitan, S. Pallua, and P. Prester, "Horizon conformal entropy in Gauss-Bonnet gravity," Physics Letters B, vol. 546, no. 1-2, pp. 119-125, 2002.

[32] A. Giacomini and N. Pinamonti, "Black hole entropy from classical Liouville theory," Journal of High Energy Physics, no. 2, 2003.

[33] N. Pinamonti and L. Vanzo, "Central charges and boundary fields for two-dimensional dilatonic black holes," Physical Review D, vol. 69, no. 8, Article ID 084012, 2004.

[34] A. J. M. Medved, D. Martin, and M. Visser, "Dirty black holes: spacetime geometry and near-horizon symmetries," Classical and Quantum Gravity, vol. 21, no. 13, pp. 3111-3125, 2004.

[35] G. Kang, J.-I. Koga, and M.-I. Park, "Near-horizon conformal symmetry and black hole entropy in any dimension," Physical Review D, vol. 70, no. 2, Article ID 024005, 2004.

[36] A. Giacomini, "Poisson algebra of diffeomorphism generators in a spacetime containing a bifurcation," Physical Review D, vol. 70, Article ID 44005, 2004.

[37] S. Carlip, "Horizon constraints and black-hole entropy," Classical and Quantum Gravity, vol. 22, no. 7, pp. 1303-1311, 2005.

[38] S. Carlip, "Conformal field theory, $(2+1)$-dimensional gravity and the BTZ black hole," Classical and Quantum Gravity, vol. 22, no. 12, pp. R85-R123, 2005.

[39] S. Carlip, "Black hole thermodynamics from euclidean horizon constraints," Physical Review Letters, vol. 99, no. 2, Article ID 021301, 2007.

[40] S. Carlip, "Symmetries, horizons, and black hole entropy," General Relativity and Gravitation, vol. 39, no. 10, pp. 1519-1523, 2007.

[41] S. Carlip, "Symmetries, horizons, and black hole entropy," International Journal of Modern Physics D, vol. 17, pp. 659-664, 2008.

[42] L.-M. Cao, Y. Matsuo, T. Tsukioka, and C.-M. Yoo, "Conformal symmetry for rotating D-branes," Physics Letters B, vol. 679, no. 4, pp. 390-395, 2009.
[43] L. Rodriguez and T. Yildirim, "Entropy and temperature from black-hole/near-horizon-CFT duality," Classical and Quantum Gravity, vol. 27, no. 15, Article ID 155003, 2010.

[44] B. K. Button, L. Rodriguez, and C. A. Whiting, "A near horizon CFT dual for Kerr-Newman-AdS," International Journal of Modern Physics A, vol. 26, p. 3077, 2011.

[45] H. Chung, "Dynamics of diffeomorphism degrees of freedom at a horizon," Physical Review D, vol. 83, no. 8, Article ID 084017, 2011.

[46] M. Cvitan, S. Pallua, and P. Prester, "Entropy of killing horizons from virasoro algebra in D-dimensional extended GaussBonnet gravity," Physics Letters B, vol. 555, no. 3-4, pp. 248-254, 2003.

[47] M. Cvitan, S. Pallua, and P. Prester, "Conformal entropy as a consequence of the properties of stationary Killing horizons," Physical Review D, vol. 70, no. 8, Article ID 084043, 2004.

[48] M. Cvitan and S. Pallua, "Conformal entropy for generalized gravity theories as a consequence of horizon properties," Physical Review D, vol. 71, no. 10, Article ID 104032, 2005.

[49] M.-I. Park and J. Ho, "Comment on: 'Black hole entropy from conformal field theory in any dimension," Physical Review Letters, vol. 83, no. 26, p. 5595, 1999.

[50] S. Carlip, "Reply to the comment by Park and Ho," Physical Review Letters, vol. 83, p. 5596, 1999.

[51] M.-I. Park and J. H. Yee, "Comment on "Entropy of 2D black holes from counting microstates',' Physical Review D, vol. 61, no. 8, Article ID 088501, 2000.

[52] O. Dreyer, A. Ghosh, and J. Wiśniewski, "Black hole entropy calculations based on symmetries," Classical and Quantum Gravity, vol. 18, no. 10, pp. 1929-1938, 2001.

[53] S. Silva, "Black-hole entropy and thermodynamics from symmetries," Classical and Quantum Gravity, vol. 19, no. 15, pp. 3947-3961, 2002.

[54] B. R. Majhi and T. Padmanabhan, "Noether current, horizon Virasoro algebra, and entropy," Physical Review D, vol. 85, no. 8, Article ID 084040, 2012.

[55] B. R. Majhi and T. Padmanabhan, "Noether current from the surface term of gravitational action, Virasoro algebra and horizon entropy," Physical Review D, vol. 86, Article ID 101501, 2012.

[56] T. Padmanabhan, Gravitation: Foundations and Frontiers, chapter 15, Cambridge University Press, Cambridge, UK, 2010.

[57] T. Padmanabhan, "Holographic gravity and the surface term in the Einstein-Hilbert action," Brazilian Journal of Physics, vol. 35, no. 2, pp. 362-372, 2005.

[58] A. Mukhopadhyay and T. Padmanabhan, "Holography of gravitational action functionals," Physical Review D, vol. 74, no. 12, Article ID 124023, 2006.

[59] S. Kolekar and T. Padmanabhan, "Holography in action," Physical Review D, vol. 82, Article ID 024036, 2010.

[60] T. Padmanabhan, "Structural aspects of gravitational dynamics and the emergent perspective of gravity," AIP Conference Proceedings, vol. 1483, pp. 212-238, 2012.

[61] R. M. Wald, "Black hole entropy is the Noether charge," Physical Review D, vol. 48, no. 8, pp. R3427-R3431, 1993.

[62] V. Iyer and R. M. Wald, "Some properties of the Noether charge and a proposal for dynamical black hole entropy," Physical Review D, vol. 50, no. 2, pp. 846-864, 1994.

[63] R. M. Wald and A. Zoupas, "General definition of "conserved quantities" in general relativity and other theories of gravity," Physical Review D, vol. 61, no. 8, Article ID 084027, 2000. 
[64] B. Julia and S. Silva, "Currents and superpotentials in classical gauge-invariant theories: I. Local results with applications to perfect fluids and general relativity," Classical and Quantum Gravity, vol. 15, no. 8, pp. 2173-2215, 1998.

[65] B. Julia and S. Silva, "Currents and superpotentials in classical gauge theories: II. Global aspects and the example of affine gravity," Classical and Quantum Gravity, vol. 17, no. 22, pp. 47334743, 2000.

[66] S. Silva, "Brane-world charges," Classical and Quantum Gravity, vol. 18, no. 8, pp. 1577-1591, 2001.

[67] M. C. Ashworth and S. A. Hayward, "Boundary terms and Noether current of spherical black holes," Physical Review D, vol. 60, no. 8, Article ID 084004, 1999.

[68] T. Padmanabhan, "Equipartition energy, Noether energy and boundary term in gravitational action," General Relativity and Gravitation, vol. 44, no. 10, pp. 2681-2686, 2012.

[69] K. Tanabe, T. Shiromizu, and S. Kinoshita, "Late-time symmetry near black hole horizons," Physical Review D, vol. 85, no. 2, Article ID 024048, 2012. 

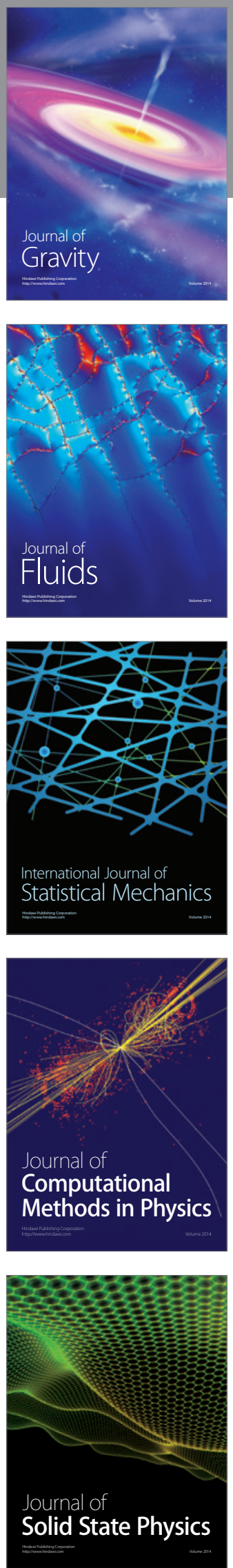

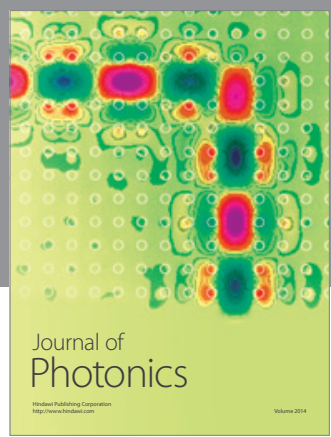

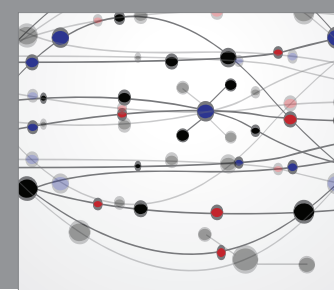

The Scientific World Journal

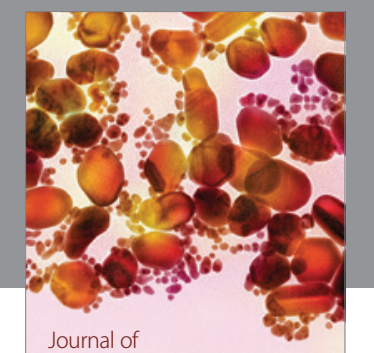

Soft Matter
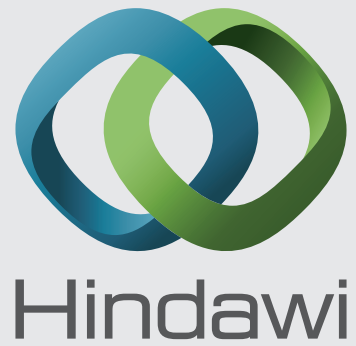

Submit your manuscripts at

http://www.hindawi.com
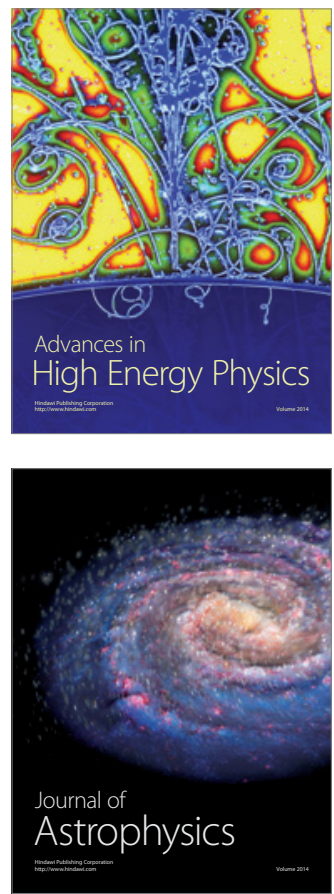
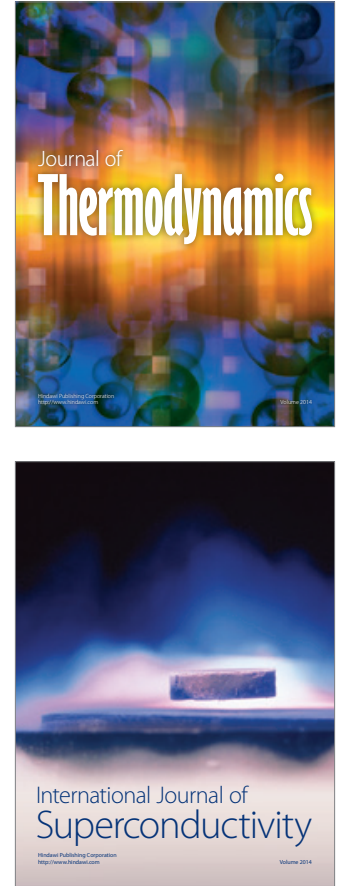
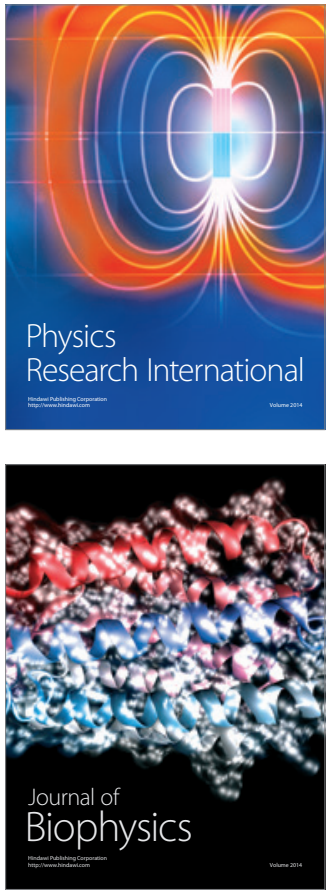
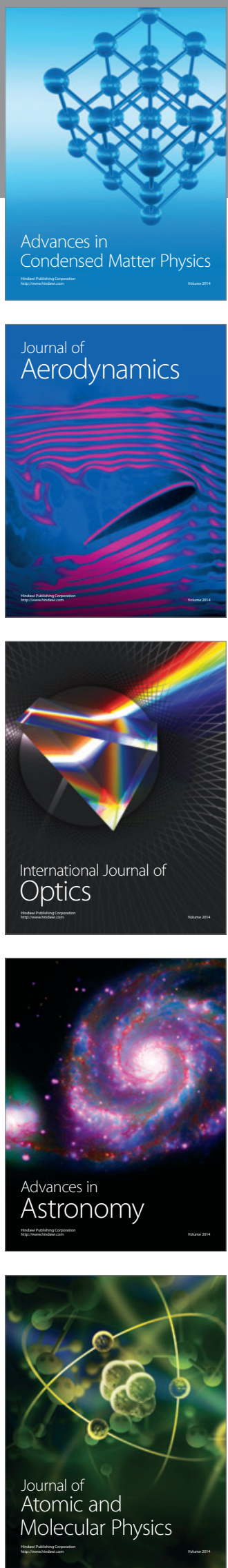